\title{
Improvement of the Performance of Transmission Holographic Grating by Hydrolysis-induced Change of the Property of Polymer Matrix
}

\author{
By Xin WANG, Yeong Hee CHO, and Yusuke KAWAKAMI
}

Trimethylsilyl ether protected 2-hydroxyethyl methacrylate was found as a quite effective component to fabricate transmission holographic polymer dispersed liquid crystal grating with high diffraction efficiency and small volume shrinkage under the irradiation of $532 \mathrm{~nm} \mathrm{Nd}$-YAG laser. Change of the property of the polymer matrix by hydrolysis of the silyl ether group enhanced by proton species produced under laser irradiation played an important role in improving the performance of the grating.

KEY WORDS: Trimethylsilyl Ether Group / Hydrolysis / Phase Separation / Diffraction Efficiency / Holographic Gratings /

Holographic polymer dispersed liquid crystal (H PDLC) formed through the anisotropic phase separation of liquid crystals from polymer matrix show potential and divers applications in flat-panel displays, ${ }^{1,2}$ switchable lenses, ${ }^{3}$ and optical switches of telecommunication, ${ }^{4}$ etc. Laser irradiation onto a homogeneous mixture of photo-polymerizable multifunctional monomer and liquid crystal (LC) results in the formation of alternating lamellae layers rich in polymer and liquid crystal through modulated polymerization kinetics. The grating morphology varies widely with the combination of LC and monomers depending on the intensity and duration of irradiation. $^{5,6}$ Precise control of lamellae domain size and distribution is crucial to optimize the overall performance of the H-PDLC gratings.

Until recently, most researches have focused on the effects of the concentration of cross-linkable multi-functional acrylate, ${ }^{7,8}$ or introduction of nanoparticles in photo-polymer systems to improve the diffraction efficiency. 9,10 However, the improvement has not been well achieved; for instance, and severe light scattering cannot be avoided even in the apparently improved nanoparticle-dispersed gratings. We reported the improved diffraction efficiency by the introduction of triethoxysilyl component in the monomer structure according to "assisted grating formation" mechanism. ${ }^{11-13}$

In this report we have focused on a novel recording system in which hydrolysable trimethylsilyl ether functional group by moisture and proton produced from initiator system was introduced to monomer structure to change the property of the polymer matrix during the recording, which facilitates the efficient phase separation of liquid crystal molecules.

\section{EXPERIMENTAL}

\footnotetext{
Materials Used in Holographic Grating Formation

Unless stated otherwise, all reagents and solvents were of commercial grade and used as received without further
}

purification. Chemical structures of components were shown in Figure 1.

2-(Trimethylsiloxy)ethyl methacrylate (TMEMA) as a vinyl monomer, trimethylolpropane triacrylate (TMPTA) as a multifunctional monomer and $N$-vinyl-2-pyrrolidinone (NVP) as a reactive diluent were purchased from Sigma Aldrich Co. In order to clearly show the effect of hydrolysis of silyl ether component on the diffraction efficiency, 2-hydroxyethyl methacrylate (HEMA), purchased from Sigma Aldrich Co., trimethylsilylmethyl methacrylate (TMMMA), purchased from Gelest Inc., were also used as vinyl monomers. Photo-initiator (PI) and photo-sensitizer (PS) system having sensitivity to visible wavelength $(\lambda=532 \mathrm{~nm})$ of Nd-YAG laser was used to generate initiating species. Diphenyliodonium hexafluorophosphate (DPI, AVOCADO research chemicals Ltd.) and 3, 3'-carbonylbis(7-diethylaminocoumarin) (KC, Kodak) were selected as PI and PS to generate cationic and radical species, respectively. The nematic LC component used here is TL 203, obtained from $\mathrm{EM}$ industries, which is composed of pentyl cyanobiphenyl (5 CB) mixed with fluoro- and chloro-substituted mesogens.

\section{Fabrication of Holographic Gratings}

The photo-polymerization solution was prepared under red light to eliminate any unexpected photo-polymerization before holographic recording. The solution for radical recording consisted of a vinyl monomer, TMPTA, NVP, DPI, KC and TL 203 in weight concentration ratio. The monomer components comprised of the relative ratio of $85 \mathrm{wt} \%$ [TMEMA (or TMMMA) + TMPTA] and $15 \mathrm{wt} \%$ NVP mixed homogenously in a glass vial. The concentrations of DPI and $\mathrm{KC}$ in the monomer components were $2 \mathrm{wt} \%$ and $0.2 \mathrm{wt} \%$, respectively. Recording solution was prepared by mixing monomer components and LC in the ratio of 65:35 by weight. A few drops of recording solution were injected into a cell between the spaces of two glass slides separated by $20 \mu \mathrm{m}$ bead spacer to control the thickness. 
<smiles>C=C(C)C(=O)OCCO[SiH3]</smiles>

2-(Trimethylsiloxy)ethyl methacrylate, TMEMA<smiles>C=C(C)C(=O)OCCO</smiles>

2-hydroxyethyl methacrylate, HEMA<smiles>C=CN1CCCC1=O</smiles>

$\mathrm{N}$-vinyl-2-pyrrolidinone, NVP<smiles>C=C(C)C(=O)OC[SiH3]</smiles>

Trimethylsilylmethyl methacrylate, TMMMA<smiles>C=CC(=O)OCC(C)(CC)COC(=O)C=C</smiles>

Trimethylolpropane triacrylate, TMPTA

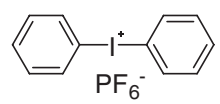

Diphenyliodonium hexafluorophosphate, DPI<smiles>CCNc1ccc2cc(C(=O)c3cc4ccc(N(CC)CC)cc4oc3=O)c(=O)oc2c1</smiles>

3, 3'-carbonylbis(7-diethylaminocoumarin), KC

Figure 1. Molecular structure and abbreviations of components for recording.

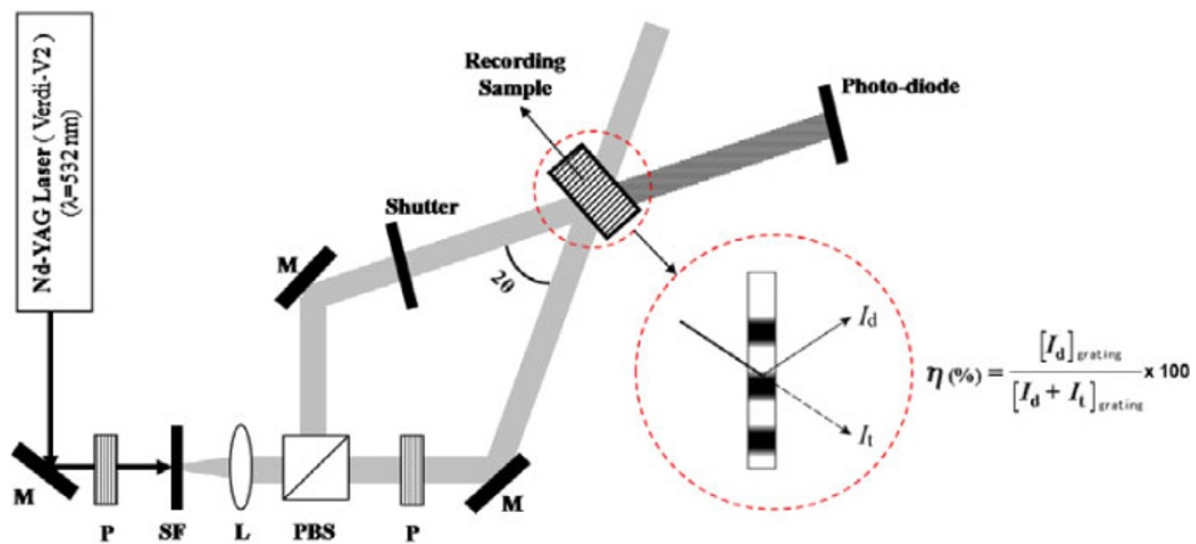

Figure 2. Experimental setup and definition of diffraction efficiency.

Holographic gratings were fabricated under $40 \mathrm{~mW} / \mathrm{cm}^{2}$ intensity, and the optimum condition was established to obtain the high diffraction efficiency, high resolution, and excellent long-term stability after recording.

\section{Optical Setup and Diffraction Efficiency of Gratings}

Nd-YAG solid-state continuous wave laser with $532 \mathrm{~nm}$ wavelength (Coherent Inc., Verdi-V2) was used as the irradiation source as shown in Figure 2. The beam was expanded and filtered by spatial filters (SF), and collimated by collimator lens (L). S-polarized beams were generated and split by controlling the two $\lambda / 2$ plates $(\mathrm{P})$ and polarizing beam splitter (PBS). Thus, separated two s-polarized beams with equal intensities were reflected by two mirrors (M) and irradiated to recording solution at a pre-determined external beam angle $\left(\theta, 16^{\circ}\right)$ which was controlled by rotating the motor-driven two mirrors and moving the rotation stage along the linear stage. The diffraction efficiency is defined as the ratio of diffraction intensity $\left[I_{\mathrm{d}}\right]$ to sum of transmitted beam intensities $\left[I_{\mathrm{d}}+I_{\mathrm{t}}\right]$ after recording, namely $\left[I_{\mathrm{d}}\right]_{\text {grating }} /$ $\left[I_{\mathrm{d}}+I_{\mathrm{t}}\right]_{\text {grating }}$.

\section{Morphology of Holographic Gratings}

Surface morphology of gratings was examined with scan- 
ning electron microscope (SEM, HITACHI, S-4500). The samples for measurement were prepared by freeze-fracturing in liquid nitrogen, and washed with methanol for $24 \mathrm{~h}$ to extract the TL 203, in case necessary. Exposed surface of the samples for SEM was coated with a very thin layer of Pt-Pd to minimize artifacts associated with sample charging (HITACHI, E-1030 ion sputter).

\section{RESULTS AND DISCUSSION}

\section{Observation of Hydrolysis of Silyl-protective Group of TMEMA by Laser Light}

Hydrolysis of TMEMA by atmospheric moisture on irradiation of the laser light was confirmed by Fourier transform infrared spectrometry (FT-IR) of the recording solution after exposure for one minute. The FT-IR showed a stretching vibration at $3419 \mathrm{~cm}^{-1}$ assignable to hydroxyl stretching band as shown in Figure 3, which indicated TMEMA could be hydrolyzed at least partially by atmospheric moisture on irradiation of $532 \mathrm{~nm}, 40 \mathrm{~mW} / \mathrm{cm}^{2}$ laser light to afford hydroxyl group.

\section{Effect of Hydrolysis on Diffraction Efficiency}

Figure 4 shows traces of real-time diffraction efficiency with different concentration ratio of TMPTA:TMMMA or TMEMA.

Figure 4(a) and 4(b) indicated gratings could be formed for both systems when the concentration of TMPTA was high. With higher concentration of TMPTA, the higher the diffraction efficiency was obtained. Almost quantitative efficiency could be attained with $35 \mathrm{wt} \%$ TMPTA. When the concentration of TMPTA decreased from 35 to $20 \mathrm{wt} \%$, the diffraction efficiency of TMMMA system decreased remarkably to almost half with longer induction time. When the concentration of TMPTA was decreased further to $10 \mathrm{wt} \%$, grating could not be formed well. The induction period became

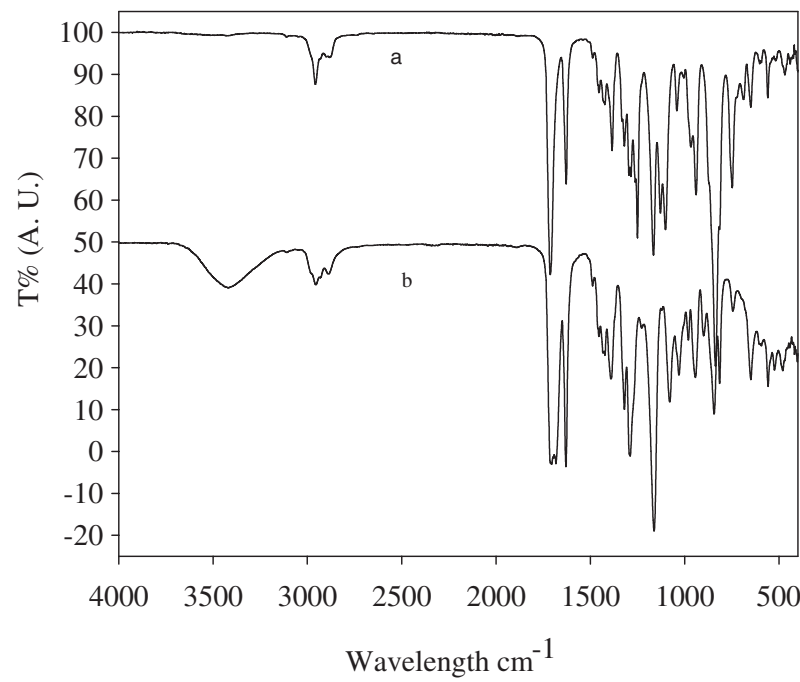

Figure 3. FT-IR spectra of (a) before and (b) after 1 minute laser irradiation. Condition: 75 wt \% TMEMA, 25 wt \% NVP, 2 wt \% PI and 0.2 wt $\%$ PS.

longer when the concentration of TMPTA was decreased, and the diffraction efficiency was decreased. The reason for this phenomenon is that the concentration of TMPTA is too low to provoke enough cross-linking and more time is needed to form the cross-linked polymer. Fifteen wt $\%$ of TMPTA seems to be the minimum concentration to form gratings in this recording system.

In TMEMA system, almost quantitative diffraction efficiency was obtained with $35 \mathrm{wt} \%$ TMPTA with a little shorter induction period than the case of TMMMA. The almost quantitative diffraction efficiency also decreased with the decrease in the concentration of TMPTA. However, rather a little change in diffraction efficiency was observed even when the concentration was decreased to $20 \mathrm{wt} \%$, and the induction

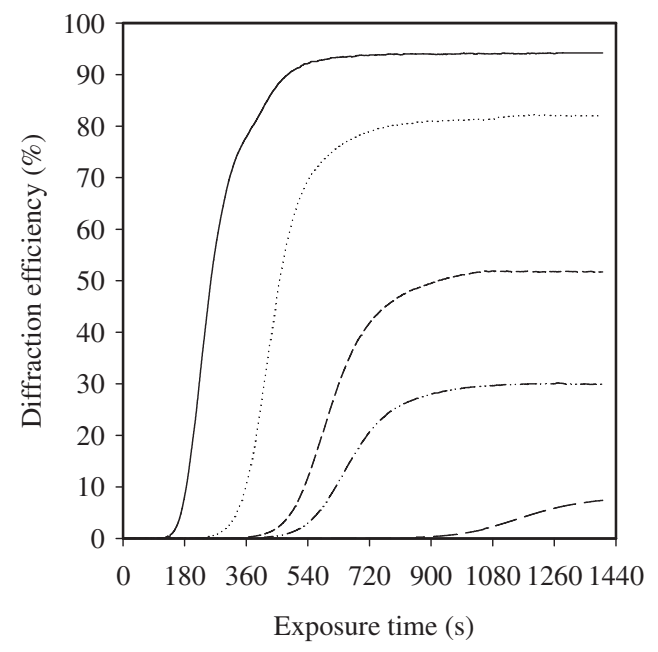

(a)

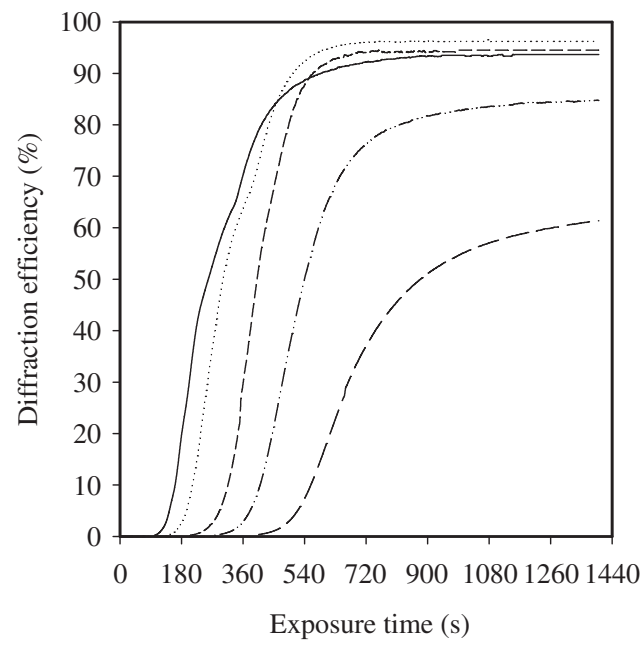

(b)

Figure 4. Real-time diffraction efficiency of gratings at different concentration of TMPTA. Condition: 85 wt $\%$ [TMMMA (or TMEMA) + TMPTA], 15 wt $\%$ NVP,

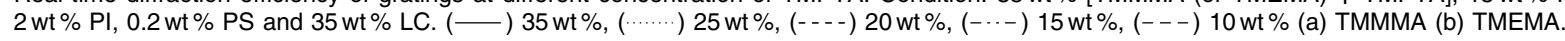


period was also much shorter than the case of TMMMA. Clear gratings could be formed even with 15 or $10 \mathrm{wt} \%$ TMPTA, although the induction time also became longer, but not significantly compared with the case of TMMMA. Nevertheless, it should be noted that the diffraction efficiency was always higher for TMEMA system with any concentration of TMPTA. Although the multi-functional acrylate TMPTA played a primary role in forming grating, hydrolysis-sensitive silyl ether component also played an important role.

With high concentration of TMPTA, due to the initial fast polymerization rate, the elastic force caused by the tighter network of TMPTA can exclude LC molecules efficiently, thereby facilitate more liquid crystals to phase-separate to form grating. This can be seen for both TMMMA and TMEMA systems. When the concentration of TMPTA was decreased, network formation took place at relatively later stage of the photo-polymerization reaction. The weaker polymer network with lower cross-linking density incorporates more LC molecules in the matrix, thereby lowering the diffraction efficiency in case of TMMMA.

In TMEMA system, atmospheric moisture together with cationic species generated from photo-initiator system ${ }^{14}$ can facilitate the hydrolysis of silyl ether groups to afford hydrophilic hydroxyl groups to polymer matrix during photopolymerization, which can efficiently induce phase separation of hydrophobic LC from the polymer matrix. The diffraction efficiency of holographic grating depicted in Figure 4(b) could remain high even the concentration of TMPTA was decreased. TMMMA is a moisture stable monomer and cannot be hydrolyzed to afford hydrophilic hydroxyl group, so, this system cannot show such effect.

HEMA is used as a hydrophilic monomer to investigate the effect of such change in polarity of polymer matrix. However, holographic grating could not be formed successfully in any concentrations of TMPTA, because of the poor miscibility with hydrophobic LC even in the initial recording solution. As mentioned above, TMEMA before hydrolysis is hydrophobic and can form homogeneous photo-polymerization syrups. Therefore, HEMA monomer was not suitable for this system containing LC component.

\section{Effect of LC Concentration on Diffraction Efficiency}

Figure 5 shows the effect of LC concentrations on the diffraction efficiency of gratings.

We found that the effect of LC on the diffraction efficiency was dramatic with increase of LC concentrations from 20 to $40 \mathrm{wt} \%$. Thirty five wt \% LC was the optimum to get good gratings as reported. ${ }^{15}$ At a higher concentration, the volume fraction of LC-rich lamella increases and the LC droplets become larger and a large scattering loss will be induced. Within the LC-rich lamellae, a large amount of photopolymer exists and the index modulation is lowered accordingly.

\section{Optical Properties of Gratings}

As mentioned above, the diffraction efficiency $\eta$ is defined as $\left[I_{\mathrm{d}}\right]_{\text {grating }} /\left[I_{\mathrm{d}}+I_{\mathrm{t}}\right]_{\text {grating }}$, namely the ratio of diffraction

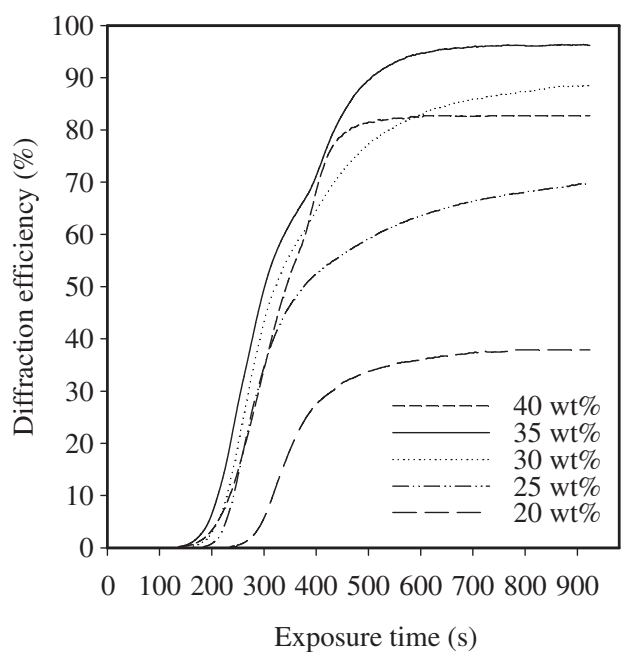

Figure 5. Real-time diffraction efficiency of gratings with 60 wt $\%$ TMEMA, 25 wt $\%$ TMPTA, 15 wt $\%$ NVP, 2 wt $\%$ PI and 0.2 wt $\%$ PS at different concentrations of LC.

Table I. Optical transmittance of grating

\begin{tabular}{|c|c|c|c|}
\hline \multirow{2}{*}{ Recording system } & \multicolumn{2}{|c|}{$\begin{array}{l}\text { Diffraction efficiency } \\
(\%)\end{array}$} & \multirow{2}{*}{$\begin{array}{l}\text { Optical transmittance }(\% \\
\qquad\left(\eta^{*} / \eta \times 100\right)\end{array}$} \\
\hline & $\eta^{*}$ & $\eta$ & \\
\hline TMEMA & 89.2 & 93.4 & 95.5 \\
\hline TMMMA & 88.6 & 94.0 & 94.3 \\
\hline
\end{tabular}

$\eta^{*}$ : Diffraction efficiency defined as $\left[I_{\mathrm{d}}\right]_{\text {grating }} /\left[I_{\mathrm{d}}+I_{\mathrm{t}}\right]_{\text {recording solution }}$. $\eta$ : Diffraction efficiency defined as $\left[I_{d}\right]_{\text {grating }} /\left[l_{d}+l_{t}\right]_{\text {grating }}$.

intensity to total transmitted intensity of the grating. The loss factors, such as absorption and scattering are ignored. To evaluate such loss factors, diffraction efficiency $\eta^{*}$ was also defined as $\left[I_{\mathrm{d}}\right]_{\text {grating }} /\left[I_{\mathrm{d}}+I_{\mathrm{t}}\right]_{\text {recording solution }}$, and the results are also included in Table I. From these results, it is obvious that the values of $\eta$ and $\eta^{*}$ are similar for two systems, indicating that there is only negligible light loss due to the Rayleigh scattering or absorption.

Optical transmittance of grating is defined as $\left[I_{\mathrm{d}}+I_{\mathrm{t}}\right]_{\text {grating }} /$ $\left[I_{\mathrm{d}}+I_{\mathrm{t}}\right]_{\text {recording solution }}=\eta^{*} / \eta$, namely as the ratio of total transmitted intensity after and before recording, which also excludes absorption and scattering, and the values of the formed gratings are listed in Table I. For the two gratings, very high optical transmittance over $90 \%$ was obtained.

\section{Scanning Electron Microscope (SEM) Photograph of Grat- ings}

To study the degree of phase separation of LC from polymer matrix, SEM measurement was carried out. Figure 6 represents the SEM photograph of gratings formed with TMEMA system after washing away the LC. The darker regions of the photograph are representative of the original location of the liquid crystals. The grating spacing is about $0.75 \mu \mathrm{m}$, which is reasonably close to the theoretical value of $0.96 \mu \mathrm{m}$ according to Bragg's law (grating spacing $\Lambda=\lambda / 2 \sin \theta, \lambda$ is $532 \mathrm{~nm}$ and $\theta$ is $16^{\circ}$ in this experiment). This result also supports the 

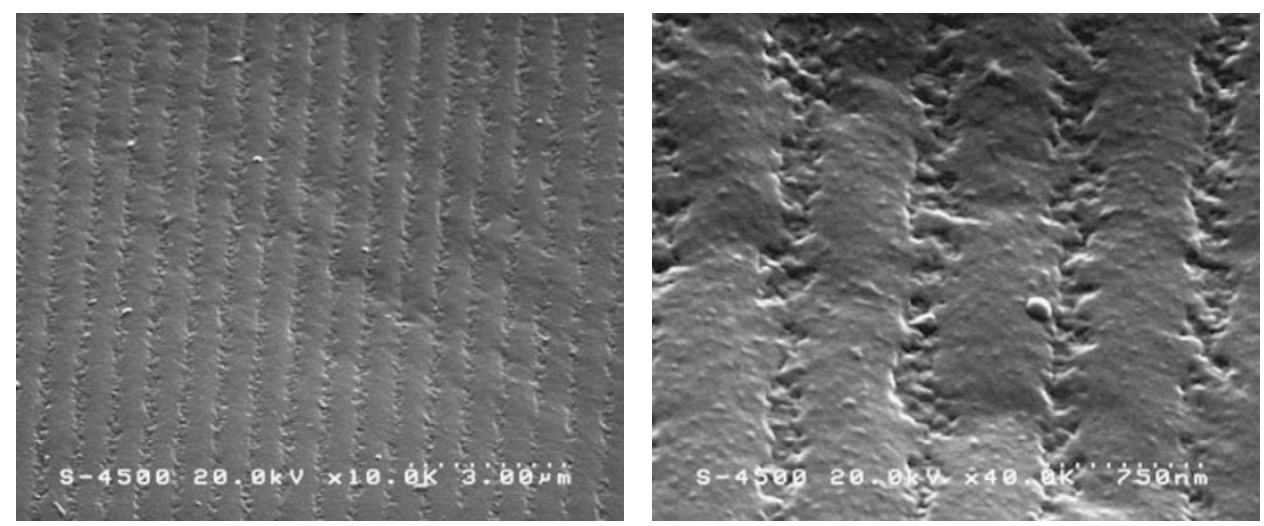

Figure 6. SEM photograph of grating formed with 70 wt $\%$ TMEMA, 15 wt $\%$ TMPTA, 15 wt $\%$ NVP, 2 wt $\%$ PI, 0.2 wt $\%$ PS and 35 wt $\%$ LC.

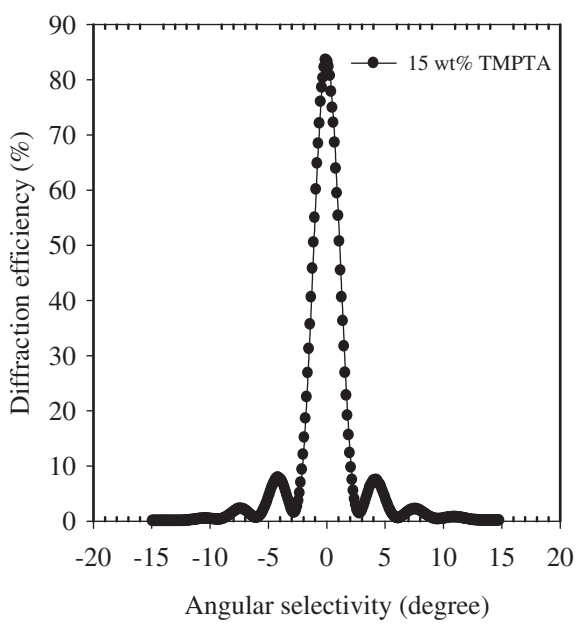

(a)

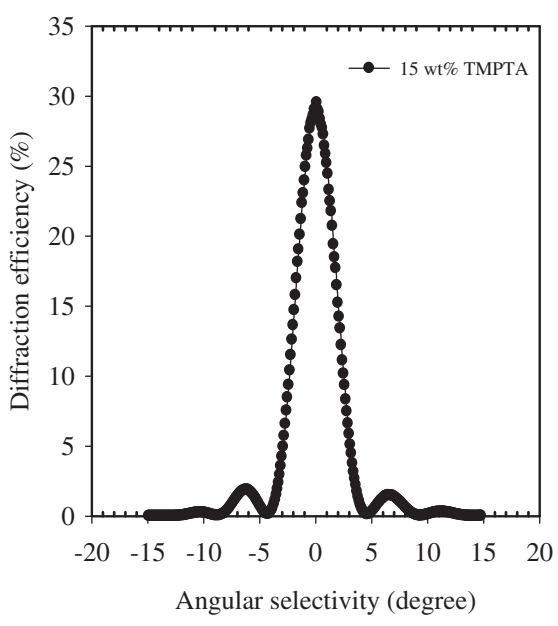

(b)

Figure 7. Angular selectivity of gratings with 70 wt $\%$ TMEMA (or TMMMA), 15 wt $\%$ TMPTA, 15 wt $\%$ NVP, 2.0 wt $\%$ PI, 0.2 wt $\%$ PS and 35 wt $\%$ LC (a) TMEMA (b) TMMMA.

minimum concentration of TMPTA is at around $15 \mathrm{wt} \%$, or higher for the formation of good grating in TMEMA system.

\section{Angular Selectivity of Gratings}

When the multiplex hologram is to be recorded it is very important to have narrow angular selectivity. The smaller the value, the more multiplex data or gratings can be recorded. Angular selectivity (from 0 degree of Bragg matching angle to first null point, $\Delta \theta_{\text {ang }}$ ) is defined by Kogelnik's coupled wave theory, ${ }^{16}$ which is given by following eq (1) and (2).

$$
\begin{gathered}
\eta=\sin ^{2}(\pi \Delta n T / \lambda \cos \theta) \\
\Delta \theta_{\text {ang }}=\left[(\lambda / T)^{2}-(\Delta n / \cos \theta)^{2}\right]^{1 / 2} / 2 n \sin \theta
\end{gathered}
$$

Where $\eta$ is the maximum diffraction efficiency, $n$ is the average refractive index of recording solution, $\Delta n$ is the modulation of refractive index of the recording medium after recording, $T$ is the thickness of the hologram, $\lambda$ is the recording wavelength, and $\theta$ is the half-angle of internal incident beams. By ignoring the very small $\Delta n$ value, the above equation could be simplified as eq (3).

$$
\Delta \theta_{\text {ang }}=\lambda / 2 n T \sin \theta
$$

By Snell's law, ${ }^{17}$ at the air interface, $n \sin \theta$ is equal to $\sin \theta_{\mathrm{ex}}$ ( $\theta_{\mathrm{ex}}$ is the external incident beam angle, $\left.n=1\right)$, the equation can be further simplified as eq (4).

$$
\Delta \theta_{\mathrm{ang}}=\lambda / 2 T \sin \theta_{\mathrm{ex}}
$$

In this research, $T, \lambda$ and $\theta_{\mathrm{ex}}$ were fixed as $20 \mu \mathrm{m}, 532 \mathrm{~nm}$ and $16^{\circ}$ respectively, angular selectivity of all the samples should be the same $2.76^{\circ}$ in theory. Figure 7 showed the angular selectivity of TMEMA and TMMMA systems with low concentrations of TMPTA. Compared with Figure 7(b), Figure 7 (a) $\left(\Delta \theta_{\text {ang }}, 2.9^{\circ}\right)$ showed only a little deviation from the theoretical values by Kogelnik's coupled wave theory, which may be attributed to the slight thickness reduction by small volume shrinkage although still existing. The reason for the low volume shrinkage is not clear at present. The repulsion force at the interface of homogenously dispersed hydrophilic segments, which were produced by hydrolysis from originally homogenously distributed as trimethylsilyl protected hydrophobic segments, in hydrophobic matrix might be a possible 
reason. More detailed study on direct observation is needed. Since this study was carried out to show the importance of the new principle to prepare grating with high diffraction efficiency and was not optimized, the temporal stability was not high.

\section{CONCLUSIONS}

Methacrylate monomer with silyl ether group was used as a component to fabricate a transmission grating of holographic polymer dispersed liquid crystal with high diffraction efficiency and small volume shrinkage. Hydrolysis-sensitive silyl ether group played an important role in improving the diffraction efficiency and volume shrinkage. 2-(Trimethylsiloxy)ethyl methacrylate photo-polymer system should be promising to fabricate a recording medium for high-density data storage device.

Acknowledgment. This work was partly supported by a Grant-in-Aid for Scientific Research (16205016) from the Ministry of Education, Science, Sports, Culture and Technology, Japan. This work was also partly supported by a City Area Program from the Ministry of Education, Science, Sports, Culture and Technology, Japan.

Received: February 5, 2008

Accepted: March 20, 2008

Published: May 14, 2008

\section{REFERENCES}

1. T. J. Trout, J. J. Schmieg, W. J. Gambogi, and A. M. Weber, $A d v$. Mater., 10, 1219 (1998).

2. T. J. Bunning, L. V. Natarajan, R. L. Sutherland, and V. P. Tondiglia, SID Digest Technol. Pap., 31, 121 (2000).

3. L. H. Domash, Y. M. Chen, B. N. Gomatam, C. M. Gozewski, R. L. Sutherland, L. V. Natarajan, V. P. Tondiglia, T. J. Bunning, and W. W. Adams, Proc. SPIE, 2689, 188 (1996).

4. L. H. Domash, Y. M. Chen, C. Gozewski, P. Haugsjaa, and M. Oren, Proc. SPIE, 3010, 214 (1997).

5. "Liquid Crystals in Confined Geometries Formed by Porous and Polymer Networks," G. P. Crawford and S. Zumer, Ed., Taylor and Francis, London, 1996

6. Y. H. Cho, C. W. Shin, N. Kim, B. K. Kim, and Y. Kawakami, Chem. Mater., 17, 6263 (2005).

7. R. T. Pogue, L. V. Natarajan, S. A. Siwecki, V. P. Tondiglia, R. L. Sutherland, and T. J. Bunning, Polymer, 41, 733 (2000).

8. M. D. Sarkar, N. L. Gill, J. B. Whitehead, and G. P. Crawford, Macromolecules, 36, 630 (2003).

9. N. Suzuki, Y. Tomita, and T. Kojima, Appl. Phys. Lett., 81, 4121 (2002).

10. N. Suzuki and Y. Tomita, Jpn. J. Appl. Phys., 42, L927 (2003).

11. Y. H. Cho and Y. Kawakami, Appl. Phys. A, 83, 365 (2006).

12. Y. H. Cho and Y. Kawakami, Silicon Chem., 3, 219 (2007).

13. Y. H. Cho, G. Suzuki, and Y. Kawakami, Polym. J., 39, 1157 (2007).

14. J. V. Crivello and F. M. Jiang, Chem. Mater., 14, 4858 (2002).

15. Y. J. Liu, X. W. Sun, H. I. Elim, and W. Ji, Appl. Phys. Lett., 90, 011109 (2007).

16. H. Kogelnik, Bell Sys. Technol. J., 48, 2909 (1969).

17. C. Oosawa, Y. Fukuta, K. Natsume, and Kometani, J. Phys. Chem., 100, 1043 (1996). 